\title{
Assessment of histopathological features of needle biopsy in recurrent prostate cancer following salvage high-intensity focused ultrasound
}

\author{
Michele Billia, MD;' Khurram M. Siddiqui, MD; Susanne Chan, MD; ${ }^{2}$ Fan Li, MD; ${ }^{1}$ Ali Al-Zahrani, MD; ${ }^{3}$ \\ Jose A. Gomez, MD;' Joseph L. Chin, MD'
}

'Department of Surgery, Division of Urologic Oncology, London Health Sciences Centre, Western University, London, ON, Canada; ${ }^{2}$ Department of Pathology and Laboratory Medicine, London Health Sciences Centre, Western University, London, ON, Canada; ${ }^{3}$ Department of Urology, University of Dammam, Dammam, Saudi Arabia

Cite as: Can Urol Assoc J 2016;10(11-12):416-22. hittp://dx.doi.org/10.5489/cuaj.3918

\section{Abstract}

Introduction: Local recurrence of prostate cancer (PCa) following radiotherapy may be treated with curative intent using salvage highintensity focused ultrasound (s-HIFU). The interpretation of needle core biopsy specimens following s-HIFU is a daunting task, even for experienced pathologists. We describe various histopathological features encountered in biopsy specimens following whole-gland s-HIFU in one of the largest descriptive studies to date.

Methods: Fifty-five patients with biopsy-proven localized radiorecurrent PCa underwent s-HIFU and transrectal ultrasound (TRUS)guided prostatic needle biopsies at 180 days post-treatment. All biopsies were reviewed by two genitourinary pathologists.

Results: PCa was detected in 11 (24\%) biopsies. Radiation therapyassociated changes were identified in all cases. Additional findings included extensive coagulative stromal necrosis (100\%), smudgy chromatin of cancer nuclei $(82 \%)$, and markedly enlarged bizarre nuclei in the residual cancer (55\%). Gleason grade assignment was possible in 10 (91\%) of these biopsies and concordance of Gleason grading between pre- and post-therapy specimens was observed in six $(60 \%)$ cases.

Conclusions: The histological interpretation of needle biopsies following salvage HIFU is challenging and requires an understanding of the histopathological changes associated with this procedure in both tumoural and non-tumoural prostatic tissue. Accurate interpretation of the morphological changes following s-HIFU is instrumental for optimization of clinical decision-making and treatment planning in recurrent PCa.

\section{Introduction}

External beam radiotherapy (EBRT) and interstitial brachytherapy (BT) are commonly used in the treatment of localized prostate cancer (PCa). ${ }^{1}$ There is evidence that $20-30 \%$ of patients fail and may benefit from local salvage therapy when systemic disease has been excluded by thorough metastatic workup. ${ }^{2,3}$
Salvage high-intensity focused ultrasound (s-HIFU) has emerged as a minimally invasive alternative, with encouraging preliminary outcomes in terms of morbidity rates and cancer control. ${ }^{4-6}$ Multiple studies have reported the morphological changes in the prostatic tissue following radiation therapy (RT) and androgen-deprivation therapy (ADT)..$^{7-9}$ In contrast, only a few reports on the histopathological changes following HIFU treatment have been published. ${ }^{10}$ The aim of this study is to perform a detailed analysis of the histopathological effects of whole-gland s-HIFU on post-treatment biopsy specimens from patients with locally recurrent PCa.

\section{Methods}

\section{Patient population}

This study includes the histopathological review of prostatic biopsy specimens (2006-2010) from patients who underwent whole-gland s-HIFU at our centre, as part of a prospectively designed trial on s-HIFU for histologically proven locally radio-recurrent $\mathrm{PCa}$ (rr-PCa). ${ }^{11,12}$ Patients' recruitment for the study was closed in 2010, whereas the followup continues as part of the prospective protocol. Screening for metastatic disease with radio-nucleotide bone scan, abdominal and pelvic computed tomography (CT) was conducted in all cases, in order to exclude patients with extraprostatic disease, who were then deemed ineligible for s-HIFU.

\section{Intervention}

HIFU was performed with the Sonoblate ${ }^{\circledR}$ system. A transrectal probe with an adjustable focal length (3 or $4 \mathrm{~cm}$ ) was employed. The HIFU protocol included treatment of the entire gland performed from side to side, subdivided into two or three zones (anterior, mid, and posterior) depending on prostate sizes, with a standard power intensity of 36-38 Watts for the anterior and mid zones (using the $4 \mathrm{~cm}$ focal 
length probe) and 20-22 Watts for the posterior zone $(3 \mathrm{~cm}$ probe). Continuous real-time transrectal monitoring of the thermal effects was performed by ultrasound visualization of the intra-prostatic acoustic pattern changes, including any "popcorn effect" (caused by collapse of gas bubbles). Special attention was paid to Denonvillier's fascia, the rectal wall, and external sphincter areas. Treatment was automatically suspended if the rectal wall temperature became excessive. The technique was standardized in all cases and performed by a single surgeon (JC). ADT, whenever initiated prior HIFU, was discontinued after the procedure in all cases.

\section{Followup}

Followup included serial prostate-specific antigen (PSA) testing at 45, 90, 180, 270, and 360 days after treatment, and every six months thereafter. Recurrence was defined according to the Phoenix criterion (PSA nadir $+2 \mathrm{ng} / \mathrm{ml}$ ), as well as by radiological, histological, and/or clinical evidence of recurrent PCa. ${ }^{13}$ Transrectal ultrasound (TRUS)-guided prostate needle biopsy was performed systematically at 180 days postoperatively, regardless of digital rectal examination (DRE) findings. Biopsies were obtained under local anesthesia using a sextant distribution scheme, as well as from sonographically and clinically suspicious areas. Patients were exempted from followup biopsy in case of refusal and/ or medical contraindications that had developed following s-HIFU, primarily recto-urethral fistulae (two cases) and recurrent urinary tract infection.

\section{Histopathological analysis}

Hematoxylin and eosin (H\&E)-stained slides prepared in the routine fashion from formalin-fixed paraffin-embedded tissue from the post-salvage HIFU prostatic biopsies were examined by two genitourinary pathologists (JAG, SC). The presence of residual cancer and high-grade prostatic intraepithelial neoplasia (PIN) was recorded and Gleason grading was performed according to the established Gleason criteria. ${ }^{12}$ The morphological features of the non-tumoural prostatic tissue were also examined. Immunohistochemical stains for a-methylacyl-CoA racemase (AMACR), p63, and high-molecular-weight cytokeratin (34-E12) were performed in selected cases on formalinfixed paraffin embedded tissue to confirm the diagnosis of PCa.

\section{Statistical analysis}

Statistical analysis was performed using PASW statistics 18 (SPSS Inc. Chicago, IL, U.S.). For comparison between groups, the Mann-Whitney $U$ test, $\chi^{2}$, and Fisher's exact test were used where appropriate. Two-sided $p$ values $<0.05$ were considered statistically significant and descriptive analyses were performed.

\section{Results}

Fifty-five patients with radio-recurrent PCa underwent salvage HIFU. Twelve men (21.8\%) received ADT, which was discontinued in all cases the day after of the procedure. The median post-salvage PSA nadir was $0.19 \mathrm{ng} / \mathrm{mL}$ (range $0.02-3.30 \mathrm{ng} / \mathrm{mL})$. Forty-six $(84 \%)$ patients underwent post-treatment biopsies. Seven patients declined biopsy, as their serum PSA levels were very low $(<0.5 \mathrm{ng} / \mathrm{mL})$ and had remained stable, and they were not keen to travel a long distance to receive the biopsy. Two patients developed recto-urethral fistulae and were exempted. Clinical characteristics of the patients are listed in Table 1. The post-treatment biopsy performed 180 days after salvage HIFU did not detect cancer in $76 \%$ of patients; however, recurrence/persistence was identified in 11 (24\%) patients; less than one-third of these had received ADT prior to initiating HIFU. The location of positive cores at biopsy was mid-base of the prostate in five cases $(45.4 \%)$, apex in four $(37 \%)$, and base in two (17.6\%). At a median followup of 25 months (range 5-56 months), no patient had died from PCa or other causes.

\section{Histopathological findings}

All post-salvage HIFU biopsy material was reviewed by two genitourinary pathologists (JAG, SC). The frequency of various histological features observed in the malignant glands and non-tumoural tissue from the post-radiation and HIFU-

\begin{tabular}{lc}
\hline $\begin{array}{l}\text { Table 1. Clinical characteristics of patients with radio } \\
\text { recurrent prostate cancer prior to salvage HIFU }\end{array}$ \\
\hline Parameters & $\begin{array}{c}\text { Pre-salvage HIFU (n=55) } \\
\text { Median age (years) }\end{array}$ \\
Median PSA (ng/ml) & 3.61 (range 57-79) \\
ADT prior s-HIFU & $12(21.8 \%$ ) \\
Gleason score, $n$ (\%) & \\
$\leq 6$ & $16(29 \%)$ \\
7 & $28(51 \%)$ \\
$\geq 8$ & $11(20 \%)$ \\
ND & - \\
No. cores at biopsy, $n$ (range) & $10(8-12)$ \\
No. positive cores, $n$ (range) & $3(1-6)$ \\
$\%$ tumour involvement, $n$ (range) & $20(2-90)$ \\
HGPIN, $n$ (\%) & $3(5)$ \\
Localization, $n$ (\%) & \\
Base & $24(44)$ \\
Mid & $8(14)$ \\
Apex & $1(2)$ \\
Mid + apex & $2(4)$ \\
Mid + apex + base & $12(22)$ \\
Mid + base & $8(14)$ \\
\hline ADT: androgen-deprivation therapy; HGPIN: high-grade prostatic intraepithelial neoplasia \\
HIFU: high-intensity focused ultrasound; s-HIFU: salvage HIFU; ND: non-determinate; PSA: \\
prostate-specific antigen. \\
\hline
\end{tabular}


Billia et al.

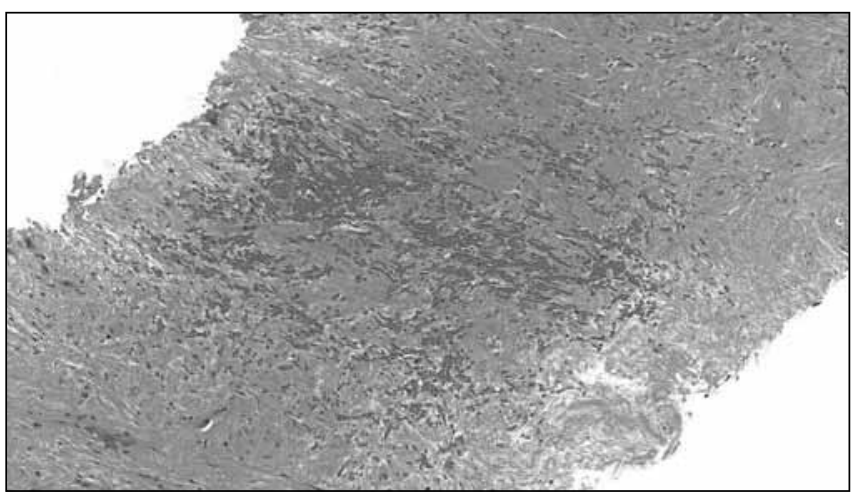

Fig.1. Histopathological features of coagulative necrosis in prostate needle biopsy.

resistant prostate biopsies is listed in Table 2. These observations are described below.

\section{Prostatic adenocarcinoma}

Malignant glands had the following histologic changes postsalvage HIFU: prominent nucleoli $(n=10)$, eosinophilic granular secretions $(n=9)$, smudgy chromatin $(n=9)$, markedly enlarged, bizarre nuclei $(n=6)$, and apoptosis of epithelial cells $(n=5)$ (Fig. 1). Gleason grading was possible in 10 of 11 biopsies. In the remaining biopsy, tissue distortion and minimal cancer volume precluded Gleason grading. Seven cases were graded as $7(3+4)$, one as $7(4+3)$, one as 8 $(4+4)$, and one as $9(4+5)$ with a tertiary pattern of 3 . We observed concordance of Gleason grading between the pre- and post-therapy biopsies in six $(60 \%)$ cases. Three $(30 \%)$ cases were upgraded and one $(10 \%)$, downgraded. The difference between the mean combined Gleason score pre-HIFU (7.214 \pm 1.050$)$ and post-salvage HIFU (7.333 \pm $0.707)$ was not statistically significant $(p=0.320)$.

\section{High-grade PIN}

High-grade PIN was identified in six post-HIFU biopsies $(55 \%)$. In five cases there was no obvious treatment effect. In only one case there were markedly enlarged, bizarre nuclei.

\section{Benign prostatic tissue}

In all cases, non-tumoural glands had variable degrees of reactive atypia $(n=11)$, atrophy $(n=11)$, and cytoplasmic eosinophilia $(n=11)$ following salvage HIFU therapy. Many of these glands were also characterized by cystic change $(n=9)$, with a minority exhibiting markedly enlarged, bizarre nuclei $(n=5)$, prominent nucleoli $(n=4)$, eosinophilic granular secretions $(n=4)$, and cytoplasmic vacuolization $(n=3)$. Other changes, including basal cell hyperplasia $(n=11)$ and squamous metaplasia $(n=8)$, were frequently observed (Fig. 2 ).

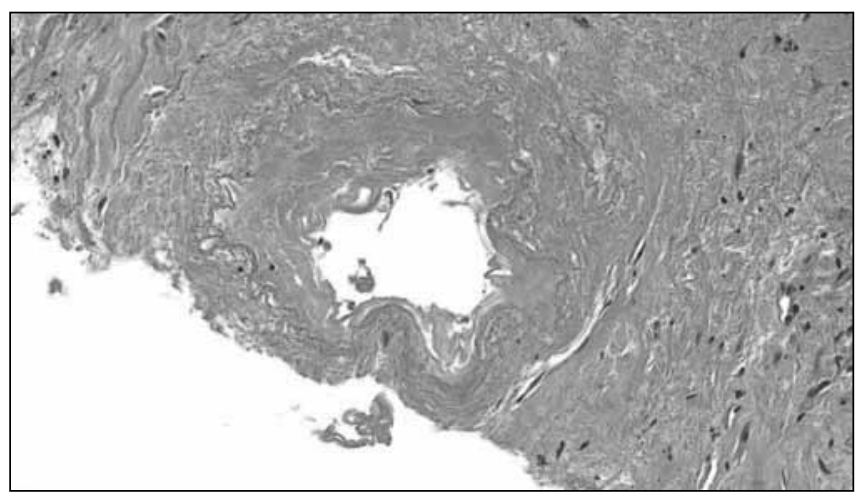

Fig 2. Histopathological features of smudgy chromatin of the adenocarcinoma nuclei, endothelial necrosis in prostate needle biopsy.

\section{Necrosis and stromal changes}

Coagulative necrosis $(n=11)$, stromal fibrosis $(n=11)$, fibroblastic atypia $(n=11)$, and stromal edema $(n=11)$ were identified in all cases. In the majority $(n=8)$, these were associated with decreased numbers of prostatic glands.

\section{Vascular changes}

Vascular changes, including vascular hyalinization $(n=11)$, vascular medial thickening $(n=10)$, collapsed lumina $(n=9)$, and endothelial necrosis $(n=7)$, were identified. In 10 specimens, extravasated red blood cells and hemosiderin deposits were also present.

\section{Inflammatory changes}

Acute and chronic inflammatory cells were present in the majority of cases with lymphocytes present most frequently $(n=10)$, followed by neutrophils $(n=9)$, plasma cells $(n=8)$, and eosinophils $(n=6)$. The appearance of these inflammatory infiltrates was non-specific.

\section{Other changes}

Nerve twig proliferation was encountered in nine cases, while calcifications were identified in three of the biopsies.

\section{Discussion}

Radical radiation therapy is one of the current options to treat localized PCa, as it offers an overall excellent cure rate. ${ }^{13}$ However, approximately one-third of patients will develop biochemical failure after radiation, as defined by the Phoenix criterion currently used in the majority of trials as the consensus definition for biochemical recurrence..$^{14,15}$

The detection of locally radio-recurrent PCa still offers the promise of cure with second-line, energy-based ablation or 


\begin{tabular}{|c|c|}
\hline \multicolumn{2}{|l|}{ Morphologic changes (\%) } \\
\hline Malignant glands & $11 / 11(100 \%)$ \\
\hline Markedly enlarged, bizarre nuclei & $6 / 11(55)$ \\
\hline Prominent nucleoli & $10 / 11(91)$ \\
\hline Smudgy chromatin & $9 / 11(82)$ \\
\hline Epithelial apoptosis & $5 / 11(45)$ \\
\hline Cytoplasmic vacuolization & $3 / 11(27)$ \\
\hline Eosinophilic granular secretions & $9 / 11(82)$ \\
\hline HPIN & $6 / 11(55 \%)$ \\
\hline No treatment effect & $5 / 6(83)$ \\
\hline Markedly enlarged, bizarre nuclei & $1 / 6(17)$ \\
\hline Benign glands & $11 / 11(100 \%)$ \\
\hline Reactive atypia & $11 / 11(100)$ \\
\hline Markedly enlarged, bizarre nuclei & $5 / 11(45)$ \\
\hline Prominent nucleoli & $4 / 11(37)$ \\
\hline Cytoplasmic eosinophilia & $11 / 11(100)$ \\
\hline Cytoplasmic vacuolization & $3 / 11(27)$ \\
\hline Atrophy & $11 / 11(100)$ \\
\hline Cystic change & $9 / 11(82)$ \\
\hline Eosinophilic granular secretions & $4 / 11(37)$ \\
\hline Basal cell hyperplasia & $11 / 11(100)$ \\
\hline Squamous metaplasia & $8 / 11(73)$ \\
\hline \multicolumn{2}{|l|}{ Necrosis } \\
\hline Coagulative necrosis & $11 / 11(100)$ \\
\hline \multicolumn{2}{|l|}{ Stromal changes } \\
\hline Fibroblastic atypia & $11 / 11(100)$ \\
\hline Fibrosis & $11 / 11(100)$ \\
\hline Edema & $11 / 11(100)$ \\
\hline \multicolumn{2}{|l|}{ Vascular changes } \\
\hline Vascular medial thickening & $10 / 11(91)$ \\
\hline Vascular hyalinization & $11 / 11(100)$ \\
\hline Endothelial necrosis & $7 / 11(64)$ \\
\hline Collapsed lumina & $9 / 11(82)$ \\
\hline Extravasated red blood bells & $10 / 11(91)$ \\
\hline Hemosiderin deposition & $10 / 11(91)$ \\
\hline \multicolumn{2}{|l|}{ Inflammation } \\
\hline Plasma cells & $8 / 11(73)$ \\
\hline Lymphocytes & $10 / 11(91)$ \\
\hline Neutrophils & $9 / 11(82)$ \\
\hline Eosinophils & $6 / 11(55)$ \\
\hline \multicolumn{2}{|l|}{ Others } \\
\hline Reduction in overall number of glands & $8 / 11(73)$ \\
\hline Nerve twig proliferation & $9 / 11(82)$ \\
\hline Calcification & $3 / 11(27)$ \\
\hline
\end{tabular}

salvage radical prostatectomy. Although the current imaging modalities are effective in ruling out the presence of obvious metastases, the sensitivity of contemporary imaging tools is still low in the detection of local recurrence. Therefore, needle biopsies are required to confirm the anatomical presence of a recurrent cancer. ${ }^{16}$

From a pathology standpoint, the interpretation of prostate needle biopsies requires experience and often the expertise of dedicated genitourinary pathologist. It is frequently challenging due to the presence of many mimics of prostatic adenocarcinoma, which can render identification of adenocarcinoma foci difficult. Such challenges are further compounded by changes associated with radiation, hormonal, or ablation therapies.

Radiation and ablation therapies, such HIFU, induce significant changes in the prostate tissue, which may present a challenge in the interpretation of needle biopsies. The difficulties in the histopathological study following salvage treatment lie in the identification of small foci of residual adenocarcinoma; the distinction of cancer from its many mimics, including atrophy, adenosis, and atypical basal cell hyperplasia; ${ }^{7}$ and the separation of treatment effects in non-tumoural tissue from recurrent or persistent adenocarcinoma. ${ }^{17}$ Moreover, biopsy cores from prostates treated with energy-based techniques are usually significantly shorter and smaller compared to the specimens retrieved from non-treated prostatic glands. Therefore, the volume of tissue in these specimens can limit the pathologist's assessment. These uncertainties can all confound the clinical management of recurrent $\mathrm{PCa}$, keeping in mind that the outcome of needle biopsy assessment is crucial in clinical decisionmaking and treatment planning.

S-HIFU has emerged as a new minimally invasive alternative to treat rr-PCa with curative intent. ${ }^{11,12}$ To date, only a few series of salvage HIFU following radiation therapy have been reported in the literature. Table 3 illustrates the functional and oncological outcomes of the recent studies, albeit with relatively short followup.

In the scenario of biochemically recurrent PCa following s-HIFU, pathological analysis of prostate needle biopsy specimens plays a crucial role in clinical management. For instance, when a biopsy report is inconclusive or not diagnostic for recurrent cancer, patients may not be considered as suitable candidates for additional treatments, such as further local therapy and/or hormonal therapy. However, if during histopathological study findings of recurrent disease are misinterpreted, this may significantly affect patent's clinical outcome. Therefore, the stakes are high for pathologists charged with the difficult task of assessing these often limited specimens from prostatic glands previously treated with radiation and HIFU.

To date, little is known about the histopathological features of prostate tissue treated with HIFU therapy. Van Leenders et al described the histology of nine radical prostatectomy specimens from patients who underwent HIFU 7-12 days before surgery. Epithelial cell damage after HIFU was analyzed with immunohistochemistry including AE1/ 
Billia et al.

Table 3. Currently published series of salvage high-intensity focused ultrasound for locally radio-recurrent prostate cancer following external beam radiation therapy and/or brachytherapy

\begin{tabular}{|c|c|c|c|c|c|c|}
\hline Author & Series (n) & $\begin{array}{l}\text { Prospective vs. } \\
\text { retrospective }\end{array}$ & $\begin{array}{l}\text { Ablation } \\
\text { system }\end{array}$ & $\begin{array}{l}\text { Median followup } \\
\text { (months) }\end{array}$ & PFS rate (year) & $\begin{array}{l}\text { Focal vs. whole- } \\
\text { gland }\end{array}$ \\
\hline Yutkin $2014^{5}$ & $19-2$ centres & Retrospective & Sonablate & $59.3(12.2-84.3)$ & $68 \%$ (4 years) & Whole-gland \\
\hline Ahmed $2012^{21}$ & 39 - single centre & Prospective & Sonablate & $17(10-29)$ & $58 \%$ (1 year) & Focal \\
\hline Udin $2012^{22}$ & $84-2$ centres & Prospective & Sonablate & $19.8(3-35.1)$ & $48 \%$ (2 years) & Whole-gland \\
\hline Berge $2011^{23}$ & 46 - single centre & Prospective & Ablatherm & $9(3-24)$ & NA & Whole-gland \\
\hline Uchida $2011^{4}$ & 22 - single centre & Prospective & Sonablate & $24(5-80)$ & $52 \%$ (5 years) & Whole-gland \\
\hline Zacharakis $2008^{24}$ & 31 - single centre & Prospective & Sonablate & $7.4(3-24)$ & $71 \%$ (NA) & Whole-gland \\
\hline Murat $2009^{25}$ & 167 - single centre & Retrospective & Ablatherm & $18.1(3-121)$ & $53 \%$ (3 years) & Whole-gland \\
\hline
\end{tabular}

AE3, CAM5.2, anti-PSA, and MIB1 antibodies. The most frequent finding was cellular damage, characterized by coagulative necrosis, as well as loss of cytokeratin-8 expression in normal epithelium, suggestive of disintegration of cellular membranes and cytoplasmic organelles. ${ }^{18}$

Biermann et al described the spectrum of morphological changes in prostatic needle biopsies obtained in 25 patients treated with whole-gland primary HIFU. In $72 \%$ of cases, necrosis was observed, often accompanied by acute, chronic, or granulomatous inflammation. Mild or moderate fibrosis was present in all biopsies. Eleven patients (44\%) had residual cancer after treatment, and in nine of the 11 patients, there was no evidence of apparent treatment effect. ${ }^{10}$

Ryan et al retrospectively analyzed the records of 45 patients who received primary HIFU, using either the Sonablate-500 or the Ablatherm system. ${ }^{19}$ Thirty patients underwent needle biopsy following HIFU either for a rising PSA (22 patients) or for a routine followup biopsy (eight patients). Seventeen biopsies (77\%) contained adenocarcinoma and in all cases pathologists were able to assign a Gleason score. When comparing pre- and post-treatment biopsies, the authors recorded $18 \%$ of cases with an upgraded Gleason score among the post-treatment needle biopsy specimens. In this series, stromal fibrosis was the commonest finding in non-tumour post-HIFU biopsy tissue (57\%), with coagulative necrosis in fewer cases $(13 \%)$.

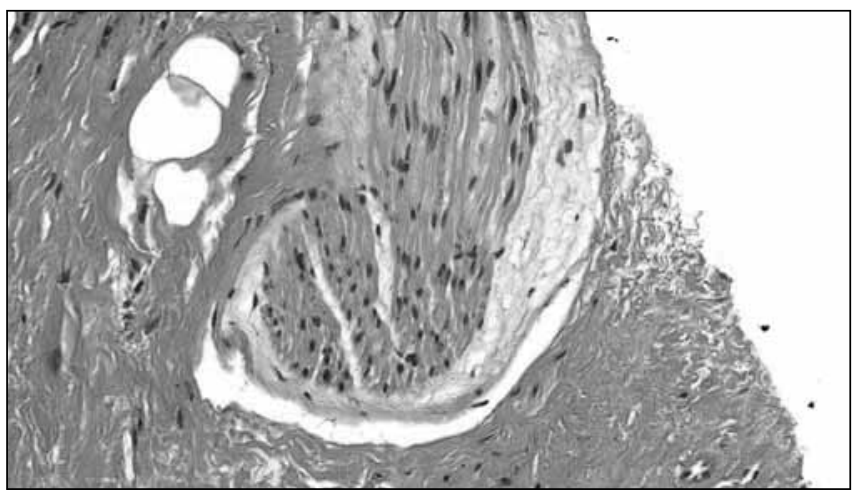

Fig 3. Histopathological features of nerve twig proliferation in prostate needle biopsy.
To our knowledge, our study is the first to report the histopathological features of needle biopsy specimens following whole-gland s-HIFU for rr-PCa. Some of our morphological findings coincide with those outlined in the above studies, mainly coagulative necrosis, stromal fibrosis, and acute/ chronic inflammation. Although our data were generated from a different population of specimens, our results are similar to those described by Biermann and Ryan on primary HIFU patients. However, in our series, we did not observe granulomatous inflammation.

Given prior history of radiation therapy in all patients, it is not surprising that many of our histopathological observations correspond to findings that have been well-characterized in the post-radiation therapy setting. ${ }^{7,8}$ These findings include the presence of prominent nucleoli, apoptosis, cytoplasmic vacuolization and eosinophilic secretions in the malignant glands, and the relative lack of treatment effect on high-grade PIN. The presence of reduced numbers of glands, reactive atypia, enlarged nuclei, prominent nucleoli, cytoplasmic eosinophilia, cytoplasmic vacuolization, and atrophy have been documented in non-tumoural glands after radiation therapy, as have been presence of eosinophilic granular secretions, basal cell hyperplasia, and squamous metaplasia. Stromal changes associated with radiation therapy were present and included fibroblastic atypia, stromal fibrosis, edema and calcification, hemosiderin deposition,

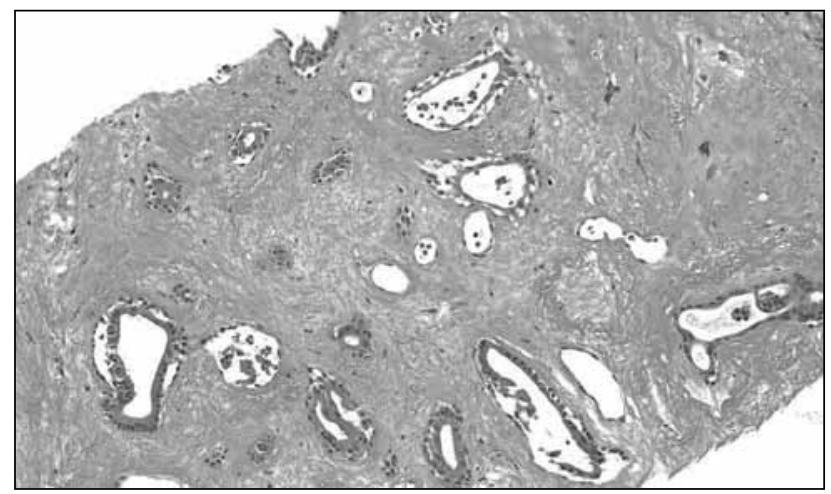

Fig 4. Histopathological features of cystic change of the non-tumoural prostatic glands in prostate needle biopsy. 
extravasated red blood cells, and non-specific inflammatory infiltrates. Vascular medial thickening, hyalinization and luminal narrowing of arteries characteristic of radiation therapy effect were also identified.

Less than one-third of the patients had received ADT prior to HIFU and this therapy was suspended following HIFU. Consequently, at the time of biopsy, these patients had been off ADT for six months or more. As in the case of radiation therapy, histopathological findings have been well-characterized in the androgen-deprivation setting. ${ }^{20}$ Reduction in numbers of neoplastic glands, apoptotic /pyknotic nuclei, and foamy vacuolated cytoplasm are hallmarks of ADT effect on tumour and were observed to a certain extent in our cases, regardless of whether the patient had received ADT or not. Discriminating between post-ADT and post-radiation therapy effect was not possible because there is some degree of morphological overlap between the two and because the morphology of prostatic adenocarcinoma a long time after the discontinuation of ADT is uncertain.

Some of our findings are less clearly associated with radiation therapy or ADT and may represent changes induced by the HIFU therapy proper. These include consistent and extensive presence of coagulative necrosis (Fig. 1), presence of smudgy chromatin of the adenocarcinoma nuclei, endothelial necrosis (Fig. 2), nerve twig proliferation (Fig. 3), markedly enlarged bizarre nuclei both in the residual cancer and high-grade PIN, and cystic change of the non-tumoural prostatic glands (Fig. 4).

With regards to the needle biopsy specimens harbouring recurrent cancer (24\%), we observed that in up to $37 \%$ of cases the tumour was located in the prostatic apex. This finding may be partially explained by intentional undertargeting of this region during pre-treatment planning, in an attempt to avoid any iatrogenic infringement on the external urethral sphincter mechanism.

Finally, we observed a concordance of Gleason grading between the pre- and post-therapy biopsies in six (60\%) cases, while three cases and one case were upgraded and downgraded, respectively. This data differ from those of Ryan et al, who reported $18 \%$ upgrading. However, our results could be biased by the selection of a population of patients at higher risk and with more aggressive disease previously treated with radiation.

Our study has limitations, including the fact that it is a retrospective pathology analysis of a relatively small series of patients in a prospective study on s-HIFU. Additionally, the overlapping effects of prior therapeutic modalities on tumoural and non-tumoural tissue make it difficult to isolate and identify morphological changes corresponding to HIFU. Nevertheless, our data provide a significant contribution to the understanding of the complex histopathology of prostatic tissue, both benign and malignant, that has undergone both radiation therapy and HIFU.

\section{Conclusion}

The management of locally recurrent PCa following s-HIFU is often a challenging task for urologists. In this scenario, needle biopsies are required to confirm the histologic presence of the cancer. Nevertheless, the histological interpretation of such biopsies is challenging and requires an understanding of the histopathological changes associated with this procedure in both tumoural and non-tumoural prostatic tissue. Therefore, knowledge of the histological features in prostatic biopsies following salvage HIFU is essential for optimizing the clinical decision-making and treatment-planning of radio-recurrent PCa.

Competing interests: Dr. Chin has been an advisor for US-HIFU and Profound Medical Inc. The remaining authors report no competing personal or financial interests.

This paper has been peer-reviewed.

\section{References}

1. D'Amico AV, Whittington R, Malkowicz SB, et al. Biochemical outcome after radical prostatectomy, external beam radiation therapy, or intersitial radiation therapy for clinically localized prostate cancer. JAMA 1998; 280:969-74. https://doi.org/10.1001/jama.280.11.969

2. Shipley WU, Thames HD, Sandler HM, et al. Radiation therapy for clinically localized prostate cancer: A multi-institutional pooled analysis. JAMA 1999; 281:598-604. https://doi.org/10.1001/ jama.281.17.1598

3. Kuban DA, Thames HD, Levy LB, et al. Long-term multi-institutional analysis of stage Tl-T2 prostate cancer treated with radiotherapy in the PSA era. Int I Radiat Oncol Biol Phys 2003;57:915-28. https://doi. org/10.1016/S0360-3016(03)00632-1

4. Uchida T, Shoii S, Nakano M, et al. High-intensity focused ultrasound as salvage therapy for patients with recurrent prostate cancer after external beam radiation, brachytherapy or proton therapy. BJU Int 2011; 107:378-82. https://doi.org/10.1111/i.1464-410X.2010.09518.x

5. Yutkin V, Ahmed HU, Donaldson I, et al. Salvage high-intensity focused ultrasound for patients with recurrent prostate cancer after brachytherapy. Urology 2014; 84:1157-62. https://doi.org/10.1016/i. urology.2014.06.054

6. Koch MO, Gardner T, Cheng $L$, et al. Phase $1 / 2$ trial of high-intensity focused ultrasound for the treatment of previously untreated localized prostate cancer. J Urol 2007;178:2366-71. https://doi. org/10.1016/i.juro.2007.08.014

7. Bostwick DG, Egbert BM, Fajardo LF. Radiation injury of the normal and neoplastic prostate. Am I Surg Pathol 1982; 6:541-51. https://doi.org/10.1097/00000478-198209000-00006

8. Bostwick DG, Meiers I. Diagnosis of prostatic carcinoma after therapy. Arch. Pathol Lab Med 2007; 131:360-71.

9. Petraki CD, Skifas CP. Histopathological changes induced by therapies in the benign prostate and prostate adenocarcinoma. Histol Histopathol 2007; 22:107-18.

10. Biermann K, Montironi R, Lopez-Beltran A. Histopathological findings after treatment of prostate cancer using high-intensity focused ultrasound (HIFU). The Prostate 2010; 70:1196-200. https://doi. org/10.1002/pros.21154

11. Williams AK, Martínez CH, Chalasani V, et al. High-intensity focused ultrasound: Where are we and where to from here? Expert Rev Anticancer Ther 2010;10:33-40. https://doi.org/10.1586/era.09.162

12. Chalasani V, Martinez $\mathrm{CH}$, Lim D, et al. Salvage HIFU for recurrent prostate cancer after radiotherapy. Prostate Cancer Prostatic Dis 2009;12:124-9. hitps://doi.org/10.1038/pcan.2008.53

13. Garg V, Gu NY, Borrego ME, et al. A literature review of cost-effectiveness analyses of prostate-specific antigen test in prostate cancer screening. Expert Rev Pharmacoecon Outcomes Res 2013;13:327-42. https://doi.org/10.1586/erp.13.26 
Billia et al.

14. Abramowitz MC, Li T, Buyyounouski MK, et al. The phoenix definition of biochemical failure predicts for overall survival in patients with prostate cancer. Cancer 2008; 112:55-60. https://doi.org/10.1002/ cncr.23139

15. Roach $M$ 3rd, Hanks $G$, Thames $H \mathrm{Jr}$, et al. Defining biochemical failure following radiotherapy with or without hormonal therapy in men with clinically localized prostate cancer: Recommendations of the RTOG-ASTRO phoenix consensus conference. Int I Radiat Oncol Biol Phys 2006; 65:965-74. https:// doi.org/10.1016/i.i.jrobp.2006.04.029

16. Crook J, Malone S, Perry G, et al. Post radiotherapy prostate biopsies: What do they really mean? Results in 498 patients. Int J Radiation Oncol Biol Phys 2000; 48:355-67. https://doi.org/10.1016/ S0360-3016(00)00637-4

17. Siders DB, Lee F. Histologic changes of irradiated prostatic carcinoma diagnosed by transrectal ultrasound. Human Pathol 1992; 23:344-51. https://doi.org/10.1016/0046-8177 (92)90080-M

18. Van Leenders GJ, Beerlage HP, Ruijter ET, et al. Histopathological changes associated with high-intensity focused ultrasound (HIFU) treatment for localized adenocarcinoma of the prostate. I Clin Pathol 2000; 59:391-4. https://doi.org/10.1136/icp.53.5.391

19. Ryan P, Finelli A, Lawrentschuk N, et al. Prostatic needle biopsies following primary high-intensity focused Ultrasound (HIFU) therapy for prostatic adenocarcinoma: Histopathological features in tumour and nontumour tissue. J Clin Pathol 2012 ;65:729-34. https://doi.org/10.1136/idinpath-2011-200460

20. Civantos F, Marcial MA, Banks ER, et al. Pathology of androgen deprivation therapy in prostate carcinoma. A comparative study of 173 patients. Cancer 1995;75:1634-41. https://doi.org/10.1002/10970142(19950401)75:7<1634::AlD-CNCR2820750713>3.0.C0;2-\#

21. Ahmed HU, Cathcart P, McCartan N, et al. Focal salvage therapy for localized prostate cancer recurrence after external beam radiotherapy: A pilot study. Cancer 2012;118:4148-55. https://doi.org/10.1002/ cncr. 27394

22. Uddin Ahmed $\mathrm{H}$, Cathcart $\mathrm{P}$, et al. Whole-gland salvage high-intensity focused ultrasound therapy for localized prostate cancer recurrence after external beam radiation therapy. Cancer 2012 15; 1 18:3071-8.

23. Berge $V, B a c o E$, Dahl $A A$, et al. Health-related quality of life after salvage high-intensity focused ultrasound (HIFU) treatment for locally radiorecurrent prostate cancer. Int J Urol 2011;18:646-51. https://doi. org/10.1111/i.1442-2042.2011.02815.x

24. Zacharakis E, Ahmed HU, Ishaq A, et al. The feasibility and safety of high-intensity focused ultrasound as salvage therapy for recurrent prostate cancer following external beam radiotherapy. BJU Int 2008;102:78692. https://doi.org/10.1111/i.1464-410X.2008.07775.x

25. Murat FJ, Poissonnier L, Rabilloud $M$, et al. Mid-term results demonstrate salvage high-intensity focused ultrasound (HIFU) as an effective and acceptably morbid salvage treatment option for locally radiorecurrent prostate cancer. Eur Urol 2009; 55:640-7. https://doi.org/10.1016/i.eururo.2008.04.091

Correspondence: Dr. Joseph L. Chin, Department of Surgery, (Urology) and Oncology, London Health Sciences Centre, Western University, London, ON, Canada; Joseph.Chin@hhsc.on.ca

\section{Zoladex}

\section{Indication and clinical use:}

Zolade ${ }^{\circledast}$ is indicated for the palliative treatment of patients with hormone-dependent advanced carcinoma of the prostate (Stage M1 or Stage D2) and for use in combination with a non-steroidal antiandrogen and radiation therapy for the management of locally advanced (T3, T4) or bulky Stage T2b, T2c carcinoma of the prostate. Zoladex ${ }^{\circledR}$ can be used as adjuvant hormone therapy to external beam irradiation for patients with locally advanced prostate cancer (Stage T3-T4).

Treatment with Zolade ${ }^{\circledR}$ and a non-steroidal antiandrogen should start 8 weeks prior to initiating radiation therapy and continue until completion of the radiation therapy. The safety and effectiveness of Zoladex ${ }^{\circledR}$ in children has not been established.

\section{Contraindications:}

Hypersensitivity to goserelin/depot or any component of the container

\section{Most serious warnings and precautions:}

Osteoporosis: Assessment of osteoporosis risk and management according to clinical practice and guidelines should be considered.

Tumor flare reaction: Patients at risk of developing ureteric obstruction should be closely monitored during the first month of therapy. Patients with vertebral metastases who are thought to be at particular risk of spinal cord compression should be closely monitored during the first month of treatment.

Injection site injuries and vascular injuries: Patients should be monitored for signs or symptoms of abdominal hemorrhage. Zoladex ${ }^{\circledast}$ is not recommended in patients with low body mass index $(\mathrm{BMI}<18.5)$ or in patients who are fully anticoagulated (INR $>2$ ).

\section{Other relevant warnings and precautions:}

- Transient elevation of serum testosterone

concentrations

- Increased cardiovascular risk factors

- Induced hypogonadism

- Impaired glucose tolerance

- Anemia

- Depression (sometimes severe)

- Pituitary-gonadal suppression

- Use in children has not been established; labeling reflects safety and effectiveness in patients over 65 years of age

- Treatment requires routine monitoring, physical examinations and appropriate laboratory tests

\section{For more information:}

Please consult the Product Monograph for Zoladex ${ }^{\circledR}$ at www.azinfo.ca/zoladex/pm965 for important information relating to adverse reactions, drug interactions and dosing information. The Product Monograph is also available by calling us at 1-800-565-5877. 